ISSN: $2178-9436$

\title{
CERTIFICAÇÃO AMBIENTAL E INDICADORES DE SUSTENTABILIDADE DA AGRICULTURA
}

\author{
ENVIRONMENTAL CERTIFICATION AND AGRICULTURAL SUSTAINABILITY \\ INDICATORS
}

Fernanda de Freitas Borges ${ }^{\mathrm{I}}$

\begin{abstract}
RESUMO
O modelo de produção atual da agricultura demanda cada vez mais áreas de plantio e ocasiona a poluição e a destruição dos ecossistemas terrestres. Devido à crescente demanda por alimentos no mundo e o modo como eles são produzidos ocasiona um avanço no esgotamento dos recursos naturais. As mudanças climáticas e o risco de falta de água ameaçam as culturas e as populações mais vulneráveis, indicando um colapso na segurança alimentar e no agravamento da desigualdade e fome no mundo. Minimizar os impactos ambientais da agricultura e agregar valor para a sociedade é um dos princípios fundamentais para que se possa alcançar os objetivos de desenvolvimento sustentável. O objetivo do estudo foi analisar os indicadores oficiais brasileiros de sustentabilidade e os utilizados na aquicultura para elaborar novos indicadores que podem ser utilizados na agricultura e subsidiar as certificações ambientais. Baseado nas diretrizes do IBGE e no que está sendo desenvolvido na aquicultura, foram elaborados dez indicadores ambientais, dez sociais e sete econômicos para a agricultura. Estes indicadores refletem o uso dos recursos naturais, a emissão de resíduos, os impactos causados no ambiente, a inclusão social, a qualidade de vida da população rural e o desempenho econômico do trabalho no campo. Os indicadores propostos se alinham com os objetivos de desenvolvimento sustentável e podem contribuir tanto para mensurar a sustentabilidade dos sistemas de produção agrícola quanto para a implementação das metas de desenvolvimento sustentável aliado aos processos de certificação.
\end{abstract}

Palavras-chave: Agronegócio. Aquicultura. Desenvolvimento sustentável. Segurança alimentar. Selo verde.

\begin{abstract}
The current production model of agriculture demands more planting areas and causes the pollution and destruction of terrestrial ecosystems. Due to increase demand for food in the world and the mode in which they are produced cause an advancement in the depletion of natural resources. The climate changes and the risk lack of water threaten the cultures and the most vulnerable populations, indicating a collapse in food security and the worsening inequality and hunger in the world. Minimizing the environmental impacts of agriculture and aggregate value to society is one of the fundamental principles for achieving the sustainable development goals. The aim of the study was to analyze the official Brazilian sustainability indicators and those used in aquaculture to develop new indicators that can be used in agriculture and subsidiary as environmental certifications. Based on the IBGE guidelines and is being developed in aquaculture, ten environmental, ten social and seven economic

I Profa. Dra. da Faculdade Nilo De Stéfani (Fatec-JB) de Jaboticabal - São Paulo - Brasil. E-mail: ferfreitasborges@gmail.com
\end{abstract}

Revista Ciência \& Tecnologia. Fatec Nilo De Stéfani (Fatec-JB). Jaboticabal - SP - Brasil. v. 12, n.1, 2020. 
ISSN: 2178-9436

indicators for agriculture were developed. These indicators reflect use of natural resources, waste emissions, impacts on the environment, social inclusion, quality of life of the rural population and the economic performance at work in the field. The proposed indicators line up with the sustainable development goals and can contribute as much for measure the sustainability of agricultural production systems as for implementation the targets of the sustainable development combined with certification processes.

Keywords: Agribusiness. Aquaculture. Sustainable development. Food security. Green seal.

Data de submissão do artigo:18/08/2020.

Data de aprovação do artigo: 20/10/2020.

DOI: $10.52138 /$ citec.v12i1.76

\section{INTRODUÇÃO}

A demanda crescente por alimentos no mundo e a destruição dos ecossistemas terrestres e aquáticos traz uma preocupação em relação ao esgotamento dos recursos naturais e a sobrevivência a curto e longo prazo. Muitos pesquisadores têm se preocupado com a forma com que se utiliza os recursos, como a água, o solo e o ar, com a quantidade de resíduos lançados no ambiente e com a perda da biodiversidade. Estudos comprovam que há um esgotamento dos recursos em curso que pode ser irreversível para o planeta e as populações. As mudanças climáticas que ameaçam as culturas e o risco de falta de água apontam para um colapso na segurança alimentar e o agravamento da desigualdade e fome no mundo.

As mudanças climáticas associadas aos efeitos antrópicos podem alterar a geografia da produção agrícola do país e a magnitude sobre um sistema produtivo depende de sua vulnerabilidade aos fenômenos climáticos associados a esta mudança (Martins et al., 2010). O cenário apresentado pelo relatório do Painel Intergovernamental sobre Mudanças Climáticas (IPCC) alerta para um aumento projetado na temperatura média da superfície global de $1,5^{\circ} \mathrm{C}$, o que ocasionará efeitos como temperaturas extremas, tanto de frio quanto de calor, mudanças no ciclo da chuva e na disponibilidade de água doce, perda de biodiversidade entre outras consequências negativas. Projeta-se que os riscos relacionados ao clima para a saúde, meios de subsistência, segurança alimentar, abastecimento de água, segurança humana e crescimento econômico aumentem com o aquecimento global (IPCC, 2018).

Diante da necessidade de se implementar ações e políticas para a mitigação desses impactos nas populações mais vulneráveis, a Organização das Nações Unidas (ONU) criou a Agenda 2030 com os objetivos de desenvolvimento sustentável e as metas a serem alcançadas pelas nações. Estas metas irão estimular ações cruciais em áreas importantes para a humanidade a serem cumpridas pelos governos, sociedade civil, setor privado e cidadãos, tendo como foco principal a redução da pobreza extrema e da fome no mundo (ONU, 2015). Neste segmento a Organização das Nações Unidas para Alimentação e Agricultura (FAO) atua no combate à fome e a pobreza, promove o desenvolvimento agrícola, a melhoria da nutrição com a busca da segurança alimentar o acesso das pessoas aos alimentos necessários para uma vida saudável. Os projetos reforçam o desenvolvimento sustentável e a agricultura para aumentar a produção de alimentos preservando os recursos naturais; a organização desenvolve projetos tanto na área da agricultura quanto na aquicultura pautados na sustentabilidade (FAO, 2020). 


\section{Ciência \& Tecnologia Fatec-JB}

ISSN: 2178-9436

Neste sentido, indicar os pontos que podem ser melhorados na produção de alimentos para criar ações a fim de minimizar os impactos causados no meio ambiente e agregar valor na sociedade trazendo o desenvolvimento econômico aliado à conservação ambiental é um dos princípios fundamentais neste século, especialmente na década atual, para que se possa alcançar os objetivos de desenvolvimento sustentável. A certificação ambiental com selos verdes pode ser uma aliada no processo de reversão da agricultura convencional destrutiva para um novo conceito de produção pautado na sustentabilidade, uma vez que pode ser utilizado pelo agronegócio o qual movimenta a economia brasileira.

O objetivo do estudo foi analisar os indicadores oficiais brasileiros de sustentabilidade e os utilizados na aquicultura para apontar novos indicadores que podem ser utilizados na agricultura e subsidiar as certificações ambientais.

\section{FUNDAMENTAÇÃO TEÓRICA}

A certificação é a modalidade mais conhecida de avaliação da conformidade e consiste na atestação dos produtos, processos, sistemas ou pessoas e um dos principais e mais utilizados procedimentos de Avaliação da Conformidade no Brasil. As empresas buscam a certificação de conformidade visando a melhoria contínua da qualidade dos seus produtos, processos e serviços, beneficiando-se com o aumento da produtividade e da competitividade (INMETRO, 2007).

Para os consumidores a certificação pode ser um indicador de que o produto, processo ou serviço atende a padrões mínimos de qualidade ambiental ou sustentabilidade. A certificação ambiental de sistemas de produção pode contribuir para a melhoria na relação com o meio ambiente e a sociedade. Desta forma, a certificação de um empreendimento agrícola poderá resultar em maior competitividade e acesso aos mercados externos, agregando valor aos seus produtos e impactando positivamente toda a cadeia de produção brasileira. Algumas certificações agrícolas conhecidas ou selos verdes, como BonSucro, RenovaBIo, Etanol Mais Verde se relacionam com os Objetivos de Desenvolvimento Sustentável da ONU e outras abordam as questões de sustentabilidade no agronegócio, como UTZ, IBD e Rainforest Alliance.

Existe uma nova demanda da agricultura no Brasil e no mundo pautada na sustentabilidade. Porém existem desafios em aumentar a produtividade de alimentos e melhorar o desempenho ambiental. Programas, metas e objetivos internacionais estão previstos em vários documentos, como a Agenda 2030 idealizada pela Organização das Nações Unidas (ONU, 2015) e o programa da Organização para a Cooperação e Desenvolvimento Econômico (OCDE) para o desenvolvimento sustentável, a qual o Brasil pretende aderir no setor da agricultura. Segundo a organização é necessário melhorar a eficiência na utilização dos recursos naturais e da agricultura, bem como o manejo da terra e da água e a minimização de emissão de poluentes para alcançar a segurança alimentar global e reduzir danos à biodiversidade (OCDE, 2013; 2017).

A previsão para 2021 da OCDE-FAO indica um aumento nos preços das commodities, o que pode influir no aumento da produtividade e, consequentemente, da pressão negativa sobre os recursos naturais (OCDE, 2013). Desta forma, o Brasil deve avançar em algumas questões importantes como a eficiência das políticas de combate ao desmatamento, melhor controle da liberação de novos agrotóxicos, da reclassificação toxicológica e de usos acentuados. Segundo a FAO, 70\% do desmatamento na América Latina é de responsabilidade do agronegócio, mais fortemente influenciada pela pecuária e plantação de grãos para a exportação. O Brasil está entre os países que mais consomem agrotóxicos no mundo e possui 


\section{Ciência \& Tecnologia Fatec-JB}

ISSN: 2178-9436

um sistema de produção de alimentos que é responsável por um grande desequilíbrio ambiental e perda de biodiversidade (BRASIL, 2018).

Diante desta perspectiva, a produção sustentável de alimentos se torna essencial para que se alcance a segurança alimentar e a conservação ambiental. O desenvolvimento sustentável que consiste em satisfazer as necessidades da geração presente, sem comprometer as possibilidades das futuras gerações em satisfazer as suas próprias necessidades (CMMAD, 1988), envolve o gerenciamento dos recursos naturais, financeiros, tecnológicos e institucionais de modo a garantir a contínua satisfação das necessidades humanas a curto, médio e longo prazo.

Para uma atividade ser sustentável deve contemplar as três dimensões da sustentabilidade e, portanto, ser ambientalmente suportável, socialmente equitável e economicamente viável (Figura 1).

Figura 1 - As três dimensões da sustentabilidade (ambiental, econômica e social)

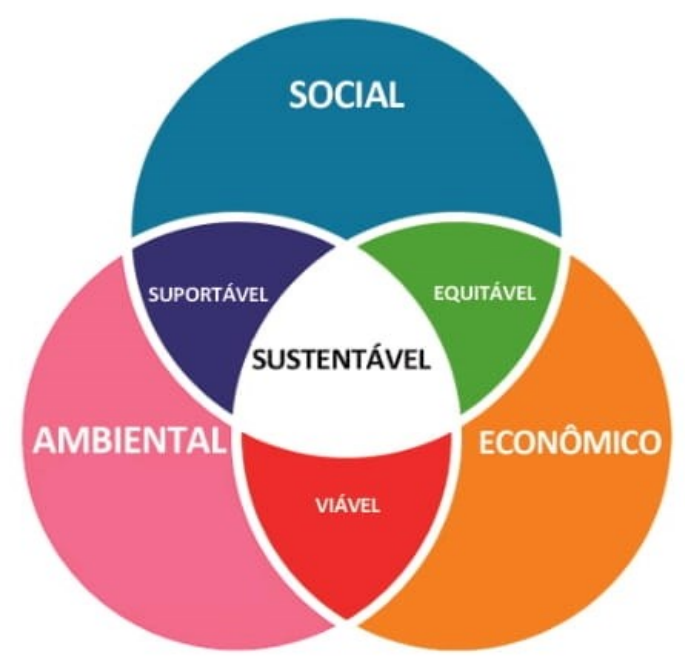

Fonte: Adaptado de Etzkowitz and Zhou (2017)

$\mathrm{Na}$ dimensão econômica, devem ser utilizadas tecnologias que minimizem os impactos ao meio ambiente com o planejamento adequado de acordo com a produção desejada. $\mathrm{Na}$ social, a atividade deve oferecer empregos para a população local, distribuindo a riqueza produzida localmente ao invés de a concentrar ou levar para outras regiões. O modo de produção deve ser harmonizado com a cultura local e hábitos da população e, consequentemente, melhorar a qualidade de vida. Ambientalmente os projetos devem ser desenvolvidos para terem harmonia com a natureza, conservando a biodiversidade e os ecossistemas do entorno. É importante incluir na avaliação as dimensões de manutenção da capacidade de suporte dos ecossistemas, a conservação da qualidade do ambiente e as dimensões socioculturais, econômicas e institucionais (VALENTI et al., 2011).

Para avaliar o quanto a atividade é sustentável pode ser utilizada a metodologia dos indicadores. Estes, são variáveis que refletem as características do sistema analisado e a sua construção é essencial no estabelecimento do método de avaliação. Os indicadores de sustentabilidade precisam apresentar relevância política, validade ou eficiência analítica, mensurabilidade e nível de agregação (OCDE, 1999). Segundo a organização os critérios de relevância política são divididos nos itens solo, água, ar, natureza, financiamento rural e sociocultural. Já a eficiência analítica se refere ao estabelecimento de ligações entre as 
ISSN: 2178-9436

atividades agrícolas e as condições ambientais. A mensurabilidade diz respeito a dados apropriados disponíveis ou métodos adequados para medir o indicador. Sendo assim, os indicadores devem ser desenvolvidos a partir de dados históricos nacionais ou regionais, que permitem evidenciar os efeitos ambientais ao longo do tempo. O nível de agregação dos indicadores determinará o nível de alcance; como propriedade, setorial, regional ou nacional, para ser aplicado em políticas públicas. Para realizar a análise, os indicadores podem ser convertidos para uma escala de desempenho e combinados para formar um índice, apresentado na forma de gráfico (KIMPARA et al., 2012).

Muitos trabalhos têm sido relatados sobre a sustentabilidade na agricultura e as formas de se avaliar seguindo a metodologia dos indicadores (FERNANDES; WOODHOUSE, 2008; RODRIGUES et al., 2010; 2016). Outras atividades agropecuárias também utilizam os indicadores como forma de avaliar seus sistemas de produção, como por exemplo na aquicultura (VALENTI, 2008; VALENTI et al., 2011, 2018).

O Instituto Brasileiro de Geografia e Estatística vem desenvolvendo indicadores de desenvolvimento sustentável conforme as organizações internacionais e adaptando-os para a realidade brasileira no que se refere às dimensões ambiental e institucional. Os indicadores são instrumentos essenciais para guiar a ação e subsidiar o acompanhamento e a avaliação do progresso alcançado rumo ao desenvolvimento sustentável e reportam-se a fenômenos de curto, médio e longo prazos (IBGE, 2015).

\section{PROCEDIMENTOS METODOLÓGICOS}

A metodologia proposta neste estudo envolve a pesquisa descritiva qualitativa, onde os indicadores propostos por outros métodos e atividade são descritos e comparados às características intrínsecas da agricultura. A partir dessa comparação são propostos novos indicadores específicos para cada dimensão da sustentabilidade.

No período de desenvolvimento do projeto de pesquisa sobre indicadores de sustentabilidade para a agricultura e tipos de certificações ambientais foi realizada uma revisão bibliográfica sistemática sobre os temas "certificação", "indicadores de sustentabilidade", "aquicultura sustentável", "impactos da agricultura" e "objetivos do desenvolvimento sustentável".

Os materiais utilizados para o desenvolvimento do estudo foram as bases online de pesquisas científicas que disponibilizam materiais, como artigos científicos, livros e boletins técnicos. Também foram consultados sites oficiais de organizações nacionais e internacionais que são referências no assunto.

\section{RESULTADOS E DISCUSSÃO}

Baseado nas diretrizes do IBGE (IBGE 2015) e no que está sendo desenvolvido na aquicultura (VALENTI et al., 2018), foram elaborados dez indicadores ambientais, dez sociais e sete econômicos para a agricultura (Quadro 1). Estes indicadores refletem o uso dos recursos naturais, a emissão de resíduos e os impactos causados no ambiente, a inclusão e a qualidade de vida da população rural e o desempenho econômico do trabalho no campo. Estes indicadores podem auxiliar em novos processos de certificações de sustentabilidade agrícola.

O Instituto Brasileiro de Geografia e Estatística (IBGE) iniciou em 2002 a trajetória de elaboração de indicadores de sustentabilidade, orientada conforme as recomendações da Comissão para o Desenvolvimento Sustentável da Organização das Nações Unidas, adaptada para a perspectiva brasileira, sendo que em 2015 houve uma nova publicação atualizada. $\mathrm{O}$

Revista Ciência \& Tecnologia. Fatec Nilo De Stéfani (Fatec-JB). Jaboticabal - SP - Brasil. v. 12, n.1, 2020. 


\section{Ciência \& Tecnologia Fatec-JB}

ISSN: 2178-9436

documento organiza os 63 indicadores em quatro dimensões; ambiental, social, econômica e institucional e, para este trabalho, serão utilizadas a título de comparação somente as três primeiras.

Quadro 1 - Indicadores das dimensões ambiental, social e econômica segundo as diretrizes governamentais e da aquicultura e os propostos para a agricultura no presente estudo

\begin{tabular}{|c|c|c|}
\hline $\begin{array}{l}\text { Diretrizes governamentais } \\
\text { (IBGE, 2015) }\end{array}$ & $\begin{array}{c}\text { Diretrizes Aquicultura (Valenti } \\
\text { et al., 2018) }\end{array}$ & $\begin{array}{c}\text { Diretrizes propostas para } \\
\text { Agricultura }\end{array}$ \\
\hline $\begin{array}{c}\text { Indicadores da Dimensão } \\
\text { Ambiental }\end{array}$ & $\begin{array}{c}\text { Indicadores da Dimensão } \\
\text { Ambiental }\end{array}$ & $\begin{array}{c}\text { Indicadores da Dimensão } \\
\text { Ambiental }\end{array}$ \\
\hline $\begin{array}{l}\text { Emissões de GEE } \\
\text { Uso de fertilizantes } \\
\text { Uso de agrotóxicos } \\
\text { Queimadas e incêndios florestais } \\
\text { Desmatamentos de biomas } \\
\text { Qualidade de água interiores } \\
\text { Espécies ameaçadas de extinção } \\
\text { Áreas protegidas } \\
\text { Espécies invasoras } \\
\text { Acesso a abastecimento de água }\end{array}$ & $\begin{array}{l}\text { Potencial de Aquecimento Global } \\
\text { Uso de fósforo } \\
\text { Uso de nitrogênio } \\
\text { Poluição Química } \\
\text { Uso de espaço } \\
\text { Uso de água } \\
\text { Potencial de Eutrofização } \\
\text { Uso de energia } \\
\text { Proporção de energia renovável } \\
\text { Risco de Espécies Criadas }\end{array}$ & $\begin{array}{l}\text { Emissão de gases poluentes } \\
\text { Uso de insumo químicos } \\
\text { Cobertura do solo } \\
\text { Erosão } \\
\text { Alterações da qualidade da água } \\
\text { Uso da água para irrigação } \\
\text { Destruição de habitats } \\
\text { Resíduos gerados } \\
\text { Redução da diversidade biológica } \\
\text { Introdução de espécies exóticas }\end{array}$ \\
\hline Indicadores da Dimensão Social & Indicadores da Dimensão Social & Indicadores da Dimensão Social \\
\hline $\begin{array}{l}\text { Rendimento domiciliar per capita } \\
\text { Rendimento médio mensal } \\
\text { Mulheres em trabalhos formais } \\
\text { Expectativa de vida } \\
\text { Prevalência de desnutrição } \\
\text { Doenças relacionadas ao } \\
\text { saneamento ambiental } \\
\text { Taxa de frequência escolar } \\
\text { Taxa de alfabetização } \\
\text { Taxa de escolaridade da população } \\
\text { adulta } \\
\text { Mortalidade por acidentes de } \\
\text { transporte }\end{array}$ & $\begin{array}{l}\text { Remuneração do trabalho por } \\
\text { unidade de produção } \\
\text { Distribuição de renda } \\
\text { Consumo local da produção } \\
\text { Desenvolvimento da economia } \\
\text { local } \\
\text { Igualdade salarial } \\
\text { Inclusão de gênero } \\
\text { Inclusão etária } \\
\text { Inclusão racial } \\
\text { Segurança do local de trabalho } \\
\text { Escolaridade }\end{array}$ & $\begin{array}{l}\text { Remuneração do produtor por } \\
\text { produção } \\
\text { Mulheres no campo } \\
\text { Remuneração das mulheres } \\
\text { Produtividade da lavoura } \\
\text { Tempo de trabalho por dia } \\
\text { Expectativa de anos trabalhados } \\
\text { Escolaridade } \\
\text { Segurança no trabalho } \\
\text { Condições de saúde } \\
\text { Mortalidade por acidentes de } \\
\text { trabalho }\end{array}$ \\
\hline $\begin{array}{l}\text { Indicadores da Dimensão } \\
\text { Econômica }\end{array}$ & $\begin{array}{l}\text { Indicadores da Dimensão } \\
\text { Econômica }\end{array}$ & $\begin{array}{l}\text { Indicadores da Dimensão } \\
\text { Econômica }\end{array}$ \\
\hline $\begin{array}{l}\text { PIB per capita } \\
\text { Taxa de investimento } \\
\text { Grau de endividamento } \\
\text { Consumo de energia per capita } \\
\text { Participação de fontes renováveis } \\
\text { de energia } \\
\text { Reciclagem }\end{array}$ & $\begin{array}{l}\text { Taxa Interna de Retorno } \\
\text { Período de Retorno de Capital } \\
\text { Relação Benefício/custo } \\
\text { Lucro líquido } \\
\text { Relação Renda Líquida } \\
\text { Investimento Inicial } \\
\text { Renda Anual }\end{array}$ & $\begin{array}{l}\text { Taxa de investimento } \\
\text { Taxa interna de retorno } \\
\text { Período de retorno do capital } \\
\text { Grau de endividamento } \\
\text { Lucro líquido } \\
\text { Renda anual } \\
\text { Logística reversa }\end{array}$ \\
\hline
\end{tabular}

Fonte: elaborado pela autora (2020)

No geral, a dimensão ambiental abrange fatores de pressão e impacto e relaciona com objetivo de preservação e conservação ambiental. Aparecem organizadas nos temas atmosfera, terra, água doce, oceanos, mares e áreas costeiras, biodiversidade e saneamento, que contemplam 19 indicadores. A dimensão social corresponde a melhoria da qualidade de vida e justiça social, abrangendo 21 indicadores nos temas população, trabalho e rendimento, 


\section{Ciência \& Tecnologia Fatec-JB}

ISSN: 2178-9436

saúde, educação, habitação e segurança, educação, distribuição da renda, equidades e condições de vida da população. Por fim, a dimensão econômica trata questões de uso e esgotamento dos recursos naturais, produção e gerenciamento de resíduos, uso de energia e desempenho macroeconômico e financeiro do país. É a dimensão que relaciona a eficiência dos processos produtivos e as alterações nas estruturas de consumo visando uma economia sustentável a longo prazo. Contempla 11 indicadores relacionados aos temas de crescimento econômico, padrões de produção e consumo dos recursos naturais (petróleo, gás natural e minerais) e gerenciamento de resíduos, como a reciclagem e rejeitos radioativos (IBGE, 2015).

$\mathrm{Na}$ aquicultura são utilizados indicadores nas três dimensões e os princípios fundamentais da sustentabilidade, são variáveis relevantes que podem ser determinadas qualitativamente e quantitativamente. Cada dimensão possui critérios específicos os quais formaram os indicadores. Eles são obtidos facilmente por todo o mundo e permitem comparar diferentes sistemas de produção em diversas regiões e com diversas espécies e escalas de tempo. Os indicadores da dimensão ambiental se relacionam aos critérios de uso de recursos naturais, a eficiência no uso dos recursos, o lançamento de poluentes no ambiente, risco de impactos na diversidade genética e biodiversidade. A dimensão social reflete a capacidade de gerar benefícios a comunidade local, distribuição justa de renda, igualdade de oportunidade e inclusão de populações vulneráveis e, a dimensão economia, mostra a eficiência no uso dos recursos financeiros, a viabilidade econômica, capacidade de resiliência e de gerar capital para reinvestimentos, além da capacidade de absorver os custos de externalidades negativas (VALENTI et al., 2018).

O modelo convencional de produção da agricultura, baseada na alta produtividade, com uso intensivo de insumos e energia ocasionou grandes impactos ambientais. No Brasil as atividades agropecuárias são responsáveis por grande parte da emissão dos gases do efeito estufa, sendo o desmatamento o principal fator devido à expansão desordenada da fronteira agrícola em áreas de vegetação nativa. Neste sentido, se destaca a produção agrícola para exportação de commodities e a emissão de gases de efeito estufa (MARTINS et al., 2010). A agricultura convencional pode causar a poluição dos mananciais com agrotóxicos e fertilizantes e acabam trazendo prejuízos para os ecossistemas e a saúde humana. Também utiliza uma grande quantidade de água para a irrigação, que corresponde a $52 \%$ do total da demanda no Brasil, com projeção de aumento de $24 \%$ até o ano de 2030 , devido a maior participação da agropecuária (ANA, 2019). Além dos impactos ambientais, muitos impactos sociais estão diretamente ou indiretamente associados aos padrões tradicionais de produção, como a desigualdade de renda, oportunidade de empregos e a subnutrição da população rural.

Os Objetivos de Desenvolvimento Sustentável propostos pela Organização das Nações Unidas são compostos por metas a serem alcançadas no prazo de quinze anos (2015-2030) e foram direcionados para a criação de uma agenda, onde cada nação deve implementar suas ações em prol do desenvolvimento sustentável, com a finalidade de eliminar por completo a insegurança alimentar. Eles preconizam, além das metas, alguns indicadores para serem alcançadas pelas nações até o ano de 2030. Com a criação da agenda cada país, estado e município deve elaborar seus próprios planos de ações de sustentabilidade (ONU, 2015).

$\mathrm{O}$ atual modelo de economia causou uma série de alterações nos ambientes naturais e consequentemente impactos negativos nos recursos hídricos, na qualidade dos solos, do ar e na biodiversidade. Com a avanço da fronteira agrícola e o aumento da mecanização no campo muitos empregos foram perdidos e os ganhos concentrados para poucos proprietários de terra. Essas novas áreas abertas enfraquecem os ecossistemas naturais e causam pobreza devido a concentração de renda. As atividades de produção de alimento, tanto a agricultura quanto a 


\section{Ciência \& Tecnologia Fatec-JB}

ISSN: 2178-9436

aquicultura, consomem ou utilizam grandes quantidades de água, que muitas vezes são fontes de poluição nos recursos hídricos. O uso da água para a produção de alimentos pode competir com outros usos, podendo causar um impacto na população, da mesma forma que a má gestão do solo. Um plano de ação desenvolvido para estas atividades no sentido de direcionar ao uso sustentável dos recursos naturais para a produção de alimentos pode contribuir para aumentar a produtividade dos sistemas e garantir a segurança alimentar sem comprometer as gerações futuras.

\section{CONSIDERAÇÕES FINAIS}

A criação destes indicadores pode dar suporte no desenvolvimento de pesquisas para a mensurabilidade da sustentabilidade dos sistemas de produção e o apontamento de melhorias sistêmicas no modelo tradicional utilizado atualmente.

Os indicadores propostos contemplam os objetivos de desenvolvimento sustentável e aliados à certificação ambiental podem direcionar o agronegócio para a sustentabilidade brasileira.

O desenvolvimento sustentável traz um novo padrão de produção e, também, de consumo, baseados na visão holística do sistema e das pessoas, melhora a condição de vida da população e preserva o meio ambiente.

\section{REFERÊNCIAS}

AGÊNCIA NACIONAL DE ÁGUAS (ANA). Estudo da ANA aponta perspectiva de aumento do uso de água no Brasil até 2030. 2019. Disponível em:

https://www.ana.gov.br/noticias/estudo-da-ana-aponta-perspectiva-de-aumento-do-uso-deagua-no-brasil-ate-2030. Acesso em: 12 de ago. 2020.

BRASIL. Ministério da Saúde. Secretaria de Vigilância em Saúde. Departamento de Vigilância em Saúde Ambiental e Saúde do Trabalhador. Agrotóxicos na ótica do Sistema Único de Saúde. Brasília: Ministério da Saúde, 2018. 2 v.

COMISSÃO MUNDIAL PARA O MEIO AMBIENTE E DESENVOLVIMENTO

(CMMAD). Nosso futuro comum. p. 10. Rio de Janeiro: Fundação Getúlio Vargas, 1987.

ETZKOWITZ, H.; ZHOU, C. The triple helix: University-industry-government innovation and entrepreneurship. New York: Routledge, 2017.

FOOD AND AGRICULTURE ORGANIZATION (FAO). Agronegócio foi responsável por quase 70\% do desmatamento na América Latina. 2016. Disponível em:

http://www.fao.org/americas/noticias/ver/pt/c/425810/. Acesso em: 06 maio 2020.

FAO no Brasil: Programas e Projetos. Disponível em:

http://www.fao.org/brasil/programas-e-projetos/es/. Acesso em: 12 ago. 2020.

FERNANDES, L. A. DE O.; WOODHOUSE, P. J. Family farm sustainability in southern Brazil: an application of agri-environmental indicators. Ecological Economics, Amsterdam, v. 66, n. 2, p. 243-257, 2008. 


\section{Ciência \& Tecnologia Fatec-JB}

ISSN: 2178-9436

INSTITUTO BRASILEIRO DE GEOGRAFIA E ESTATÍSTICA (IBGE). Indicadores de desenvolvimento sustentável: Brasil 2015. Coordenação de Recursos Naturais e Estudos Ambientais e Coordenação de Geografia. Rio de Janeiro: IBGE, 2015. 352 p.

INSTITUTO NACIONAL DE METROLOGIA, QUALIDADE E TECNOLOGIA (INMETRO). Livreto: Avaliação da Conformidade. Diretoria da Qualidade. Ed. 5, 2007. Disponível em: http://www.inmetro.gov.br/inovacao/publicacoes/acpq.pdf. Acesso em: 6 jul. 2020.

KIMPARA, J. M.; ZADJBAND, A. D.; VALENTI, W. C. Métodos para medir a sustentabilidade na aquicultura. Documentos Embrapa Meio-Norte, n. 218, 2012. 71 p.

MARTINS, S. R., SCHLINDWEIN, S. L., D'AGOSTINI, L. R., BONATTI, M., DE VASCONCELOS, A. C. F., HOFFMANN, A. F.; FANTINI, A. C. Mudanças climáticas e vulnerabilidade na agricultura: desafios para desenvolvimento de estratégias de mitigação e adaptação. Revista Brasileira de Ciências Ambientais (Online), n. 17, p. 17-27, 2010.

ORGANISATION FOR ECONOMIC CO-OPERATION AND DEVELOPMENT (OECD). Environmental indicators for agriculture: Concepts and Framework. Paris: OECD Press. 1999, v. 1, 45 p.

. Global Food Security: Challenges for the Food and Agricultural System. OECD Publishing. Compendium of Agri-environmental Indicators. 2013. Disponível em: https://www.oecd-ilibrary.org/agriculture-and-food/global-food-security_9789264195363-en. Acesso em: 06 maio 2020.

. Water Risk Hotspots for Agriculture, Studies on Water. OECD Publishing, Paris. 2017. Disponível em: https://www.oecd-ilibrary.org/agriculture-and-food/water-risk-hotspotsfor-agriculture_9789264279551-en. Acesso em: 06 de maio de 2020.

ORGANIZAÇÃO DAS NAÇÕES UNIDAS (ONU). Transformando nosso mundo: a agenda 2030 para o desenvolvimento sustentável. 2015. Disponível em: https://nacoesunidas.org/pos2015/agenda2030/. Acesso em: 09 jul. 2020.

PAINEL INTERGOVERNAMENTAL SOBRE MUDANÇAS CLIMÁTICAS (IPCC). Sumário para Formuladores de Políticas. 2018. Disponível em: https://www.ipcc.ch/site/assets/uploads/2019/07/SPM-Portuguese-version.pdf. Acesso em: 20 jul. 2020.

RODRIGUES, G. S.; RODRIGUES, I. A.; BUSCHINELLI, C. C. A.; BARROS, I. Integrated farm sustainability assessment for the environmental management of rural activities. Environmental Impact Assessment Review, Amsterdam, v. 30, n. 4, 229-239, 2010.

RODRIGUES, G. S.; PIMENTA, S. C.; CASARINI, C. R. A. Ferramentas de avaliação de impactos ambientais e indicadores de sustentabilidade na Embrapa. Boletim de Pesquisa e Desenvolvimento, n. 105. Jaguariúna: Embrapa Meio Ambiente, 2016. 21 p. 


\section{(D) Ciência \& Tecnologia Fatec-JB}

ISSN: 2178-9436

VALENTI, W. C. A Aquicultura Brasileira é Sustentável? In: IV Seminário Internacional de Aquicultura, Maricultura e Pesca. 2008, Florianópolis. Anais[...]. Santa Catarina: AQUAFAIR, 2008, p. 1-11.

VALENTI, W. C.; KIMPARA, J. M.; PRETO, B. L. Measuring Aquaculture Sustainability. World Aquaculture, v. 42, n. 3, p. 26-30, 2011.

VALENTI, W. C.; KIMPARA, J. M.; PRETO, B. D. L.; MORAES-VALENTI, P. Indicators of sustainability to assess aquaculture systems. Ecological indicators, v. 88, p. 402-413, 2018. 